\title{
Anatomia foliar de espécies de Eugenia L. (Myrtaceae) oriundas da restinga de Algodoal/Maiandeua-Pará
}

\section{Adalgisa da Silva Alvarez ${ }^{1,3}$ \& Rolf Junior Ferreira Silva ${ }^{2}$}

Enviado em setembro de 2012; aceito em novembro de 2012.

\section{Resumo}

A ampliação do número de espécies de Myrtaceae analisadas anatomicamente é necessária para se verificar a maior confiabilidade das inferências taxonômicas. Visando contribuir com dados estruturais à família Myrtaceae e ao gênero Eugenia, objetivou-se neste trabalho descrever a estrutura anatômica foliar de E. biflora (L.) DC., E. flavescens DC., E. patrisii Vahl. e E. tapacumensis Berg. ocorrentes na restinga da ilha de Algodoal/Maiandeua-Pará. São características comuns às quatro espécies examinadas: sinuosidade parietal das células epidérmicas da face adaxial da lâmina foliar; cutícula foliar espessa; tricomas tectores unicelulares; folhas hipoestomáticas; estômatos paracíticos e anomocíticos; mesofilo dorsiventral; cavidades secretoras com conteúdo reativo para lipídios, principalmente no parênquima paliçádico; idioblastos secretores com conteúdo reativo para compostos fenólicos e lipídicos; nervura central e pecíolo com feixe vascular bicolateral. A estrutura anatômica foliar das quatro espécies de Eugenia aqui estudadas apresentou características universais à família Myrtaceae.

Palavras-chave: Análise estrutural, Microquímica, Cavidade secretora.

\section{Abstract}

(Leaf anatomy of species of Eugenia L. (Myrtaceae) arising from the sandy coastal Algodoal/Maiandeua-Pará). The increase in the number of species of Myrtaceae analyzed anatomically is needed to verify the reliability of higher

\footnotetext{
DOI: http://dx.doi.org/10.5007/2178-4574.2012n41p83

1,3 Programa de Pós-Graduação em Biologia Vegetal, Universidade Federal Rural da Amazônia, Av. Presidente Tancredo Neves, 2501- Montese- CEP: 66.077-901 - Belém, PA, Brasil.

adalgisa_alvarez@yahoo.com.br

${ }^{2}$ Programa de Pós-Graduação em Botânica, Instituto Nacional de Pesquisas da Amazônia, Av. André Araújo, 2936 - Aleixo - CEP: 69.060-001 - Caixa Postal: 478 - Manaus, AM, Brasil. rolfjr@ hotmail.com ${ }^{3}$ Autor para correspondência: adalgisa_alvarez@yahoo.com.br
}

\section{(cc) $\mathrm{BY}-\mathrm{NC}$} Este artigo é de Acesso Livre, disponibilizado sob os termos da Creative Commons Attribution 3.0 Unported License (http://creativecommons.org/licenses/by/3.0/) que permite uso não-comercial, distribuição e reprodução em qualquer meio, desde que este trabalho original seja devidamente citado. 
taxonomic inferences. In order to contribute with structural data to Myrtaceae family and to Eugenia genus, the leaf anatomical structure of E. biflora (L.) DC., E. flavescens DC., E. patrisii Vahl. And E. tapacumensis Berg. arising from the sandy coastal Algodoal/Maiandeua-Pará was described. Some characteristics were irrespective of the four examined species: adaxial surface of epidermal cells with sinuous anticlinal walls; thick leaf cuticle; non-glandular unicellular trichomes; hypostomatic leaves; paracytic and anomocytic stomata; dorsiventral mesophyll; secretory cavities with positive oil content, principally at palisade parenchyma; secretory idioblasts with positive phenol and oil contents; bicollateral bundle on the midrib and petiole. The leaf anatomical structure of four examined species of Eugenia showed universal features to Myrtaceae.

Key words: Structural Analysis, Microchemistry, Secretory Cavities.

\section{Introdução}

Myrtaceae possui mais de 3.000 espécies distribuídas em cerca de 140 gêneros, tornando-a a maior família da ordem Myrtales (Johnson \& Briggs 1984, Watson \& Dallwitz 2007). No Brasil, espécies de Myrtaceae restringem-se à tribo Myrteae, subdividida classicamente em Myrtinae, Eugeniinae e Myrciinae (Lucas et al. 2005, Wilson et al. 2005).

Eugenia L. corresponde a um dos maiores e mais complexos gêneros de Myrtaceae, com grande similaridade entre os taxa, o que dificulta bastante o trabalho de especialistas da família. Inferência especial faz-se aos trabalhos de Barroso et al. (1978, 1984) quando se referem ao acúmulo de material botânico de espécies de Myrtaceae nos herbários que apresentam problemas de identificação taxonômica.

Nas áreas de restinga, gêneros como Eugenia L., Psidium L., Myrciaria O. Berg, Marlierea Cambess. e Campomanesia Ruiz \& Pav. são de grande importância botânica e econômica por fornecerem frutos comestíveis, madeira e essências aromáticas (Joly 1985, Pereira 1985).

Estudos anatômicos têm sido utilizados em análises taxonômica e evolutiva de Myrtaceae e Myrtales (Johnson \& Briggs 1984, Sajo \& Rudall 2002, Cardoso \& Sajo 2004, Leon \& Williams 2005), mas, poucos representantes da flora neotropical têm sido incluídos nestas análises, notadamente aqueles de Myrteae (Gomes et al. 2009). Diferentes taxa de Myrtaceae tiveram sua anatomia foliar examinadas e destacam-se para espécies de Eugenia os trabalhos de Behar (1971), Pereira (1985), Machado et al. (1988), Neves \& Donato (1989), Hussin et al. (1992), Khatijah et al. (1992), Fontenelle et al. (1994), Callado (1997), Jorge et al. (2000), Medeiros (2000), Cardoso \& Sajo (2004), Donato \& Morretes $(2007,2009)$ e Silveira \& Harthman (2010).

A ampliação do número de espécies de Myrtaceae analisadas anatômicamente faz-se necessária para se verificar a maior confiabilidade das inferências 
taxonômicas (Gomes et al. 2009). Visando contribuir com dados estruturais à família Myrtaceae e ao gênero Eugenia, objetivou-se neste trabalho descrever a estrutura anatômica foliar de E. biflora (L.) DC., E. flavescens DC., E. patrisii Vahl. e E. tapacumensis Berg. ocorrentes na restinga da ilha de Algodoal/Maiandeua-Pará.

\section{Material e Métodos}

\section{Localização da área de estudo}

O material botânico foi oriundo da ilha de Algodoal, do Município de Maracanã-Pará, localizada entre as coordenadas geográficas $00^{\circ} 35^{\prime} 03^{\prime \prime}$ a $00^{\circ} 38^{\prime} 29^{\prime}$ "

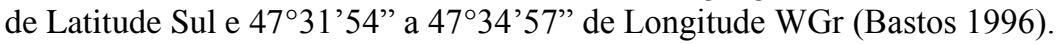

\section{Material botânico}

As amostras botânicas utilizadas para o presente estudo foram folhas adultas completamente expandidas do $4^{\circ}$ nó, coletadas de cinco espécimes por espécie. Material testemunho foi incorporado ao herbário João Murça Pires (MG) do Museu Paraense Emílio Goeldi, registrado como segue: E. biflora (L.) DC. (MG 141021), E. flavescens DC. (MG 150968), E. patrisii Vahl. (MG 149042) e E. tapacumensis Berg. (MG 141023).

\section{Métodos}

$\mathrm{O}$ estudo anatômico foi realizado em material in natura e fixado. A fixação foi feita em $\mathrm{FAA}_{50}$ (formaldeído PA, ácido acético PA, álcool etílico 50\%) por 24 horas (Johansen 1940), lavado em álcool etílico 50\% e conservado em álcool etílico $70 \%$.

Para a dissociação de epidermes, amostras das regiões do ápice, base e nervura central da lâmina foliar foram imersas em mistura de Jeffrey $10 \%$ por 24 horas (Johansen 1940).

As secções transversais da lâmina foliar, feitas nas mesmas regiões citadas à epiderme, e da região mediana do pecíolo foram feitas à mão livre, com auxilio de lâmina de aço e utilizando-se cortiço como suporte.

As epidermes dissociadas e as secções foliares obtidas foram coradas em azul de astra e fucsina básica (Braga 1977), seguida da desidratação pela série alcoólica e da pós-desidratação pela série aceto-butílica crescentes, com posterior montagem em resina sintética (Johansen 1940).

Testes microquímicos foram feitos em secções histológicas de folhas in natura. Foram utilizados ácido clorídrico 10\% (Chamberlain 1932) para verificar a presença de cristais de oxalato de cálcio; cloreto férrico a 10\% (Johansen 1940) para detectar compostos fenólicos e sudam IV (Johansen 1940) para identificar compostos lipídicos.

As fotomicrografias foram obtidas em fotomicroscopio Zeiss. 


\section{Resultados e Discussão}

A face adaxial da superfície da lâmina foliar em vista frontal, nas espécies estudadas, é formada por células poligonais com paredes anticlinais sinuosas e idioblastos. Os idioblastos correspondem às cavidades secretoras subepidérmicas, consideradas por Solereder (1908) e Metcalfe \& Chalk (1950) como um caráter taxonômico universal da família Myrtaceae (Figura 1).

A sinuosidade parietal das células epidérmicas de espécies de Eugenia foi observada por Fontenelle et al.(1994)em onze espécies deste gênero,por Pereira (1985) em E. nitida Cambess.; por Neves \& Donato (1989) em E. uniflora L. e por Donato \& Morretes (2009) em E. florida DC. Como paredes epidérmicas sinuosas têm sido verificadas em espécies de Eugenia oriunda de diferentes ecossistemas, infere-se que tal característica seja de natureza constitutiva, isto é, inerente ao genótipo, e não ecológico-adaptativa. Rocha \& Neves (2000) e Gomes et al. (2005), ao estudarem espécies de Hibiscus L.(Malvaceae) e treze espécies de Celastraceae, respectivamente, consideraram que o formato das paredes celulares epidérmicas é uma condição geneticamente fixada e não uma expressão à variação do ambiente. Premissa esta aplicável para Myrtaceae e que constitui um bom caráter taxonômico (Gomes et al. 2009).
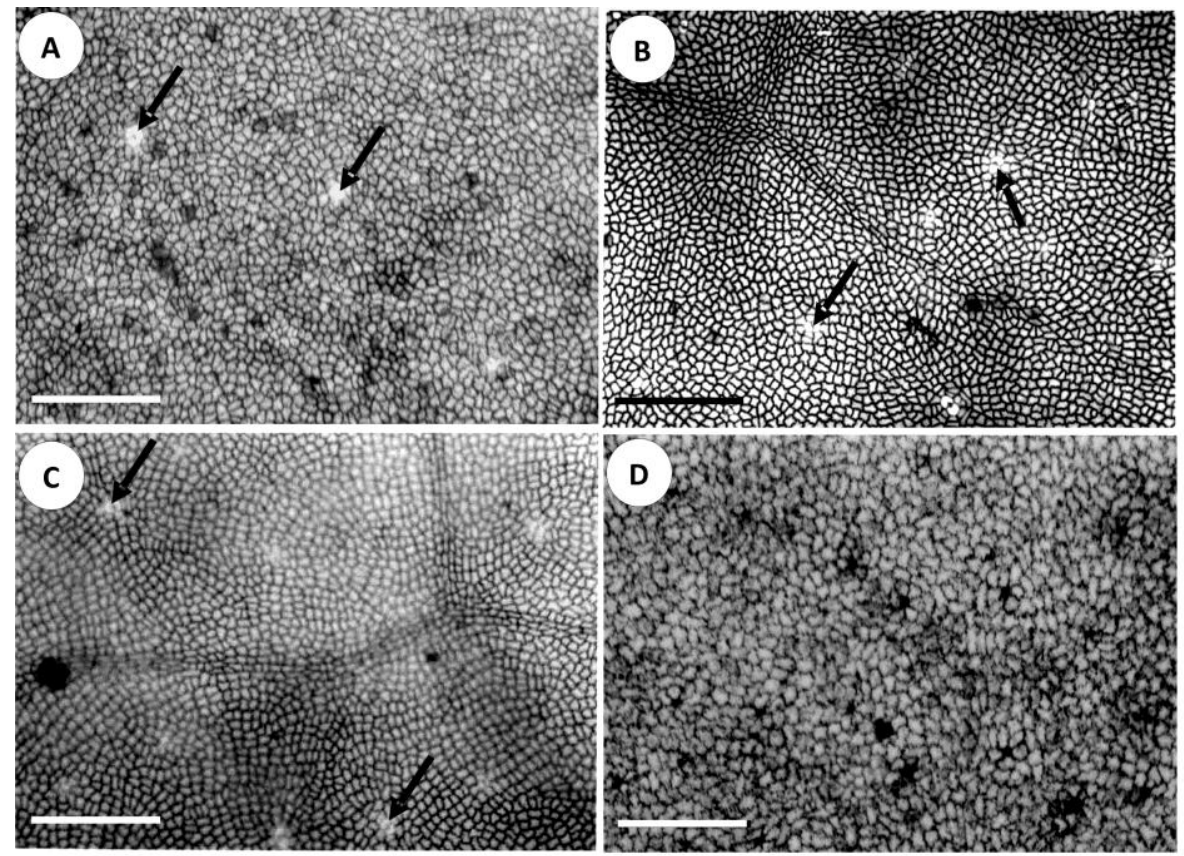

Figura 1. Vista frontal da face adaxial da superfície da lâmina foliar Eugenia L. 1A:

E. biflora (L.) DC.; 1B: E. patrisii Vahl.; 1C: E. flavescens DC.; 1D: E. tapacumensis Berg. Setas $=$ idioblastos que correspondem às cavidades secretoras subepidérmicas. Barras $=300 \mu \mathrm{m}$. 
As folhas das espécies estudadas são hipoestomáticas, com ocorrência de estômatos anomocítico e paracítico nas quatro espécies. Em E. tapacumensis e E. flavescens, ocorre ainda o tipo anisocítico (Figura 2).

Metcalfe \& Chalk (1950) citam estômatos anomocíticos como os mais comuns a Myrtaceae, embora o tipo anisocítico já tenha sido descrito para Psidium cattleyanum Sabine (Arruda \& Fontenelle 1994) e o paracítico para P. guayava Raddi (Sousa 1971, Tantawy 2004), E. uniflora L.(Auricchio \& Bacchi 2003; Tantawy 2004) e Campomanesia adamantium (Cambess.) O. Berg., Myrcia cordiifolia DC., M. decrescens O. Berg. e M. torta DC. (Gomes et al. 2009).

Tricomas tectores unicelulares simples ou apenas suas cicatrizes, como é mais comum, foram observados restritos à face abaxial da superfície da lâmina foliar das espécies de Eugenia analisadas (Figuras 2B, 2D e 3D). Tricomas em Myrtaceae são geralmente unicelulares e não ramificados (Metcalfe \& Chalk 1950), raro em poucos gêneros do velho mundo em que são pluricelulares (Johnson \& Briggs 1984), mostrando-se como um caractere relevante à identificação das espécies do Brasil (Landrum \& Kawasaki 1997), a exemplo das amazônicas de Marlierea Cambess. que foram agrupadas com base no indumento (Rosario \& Secco 2006).
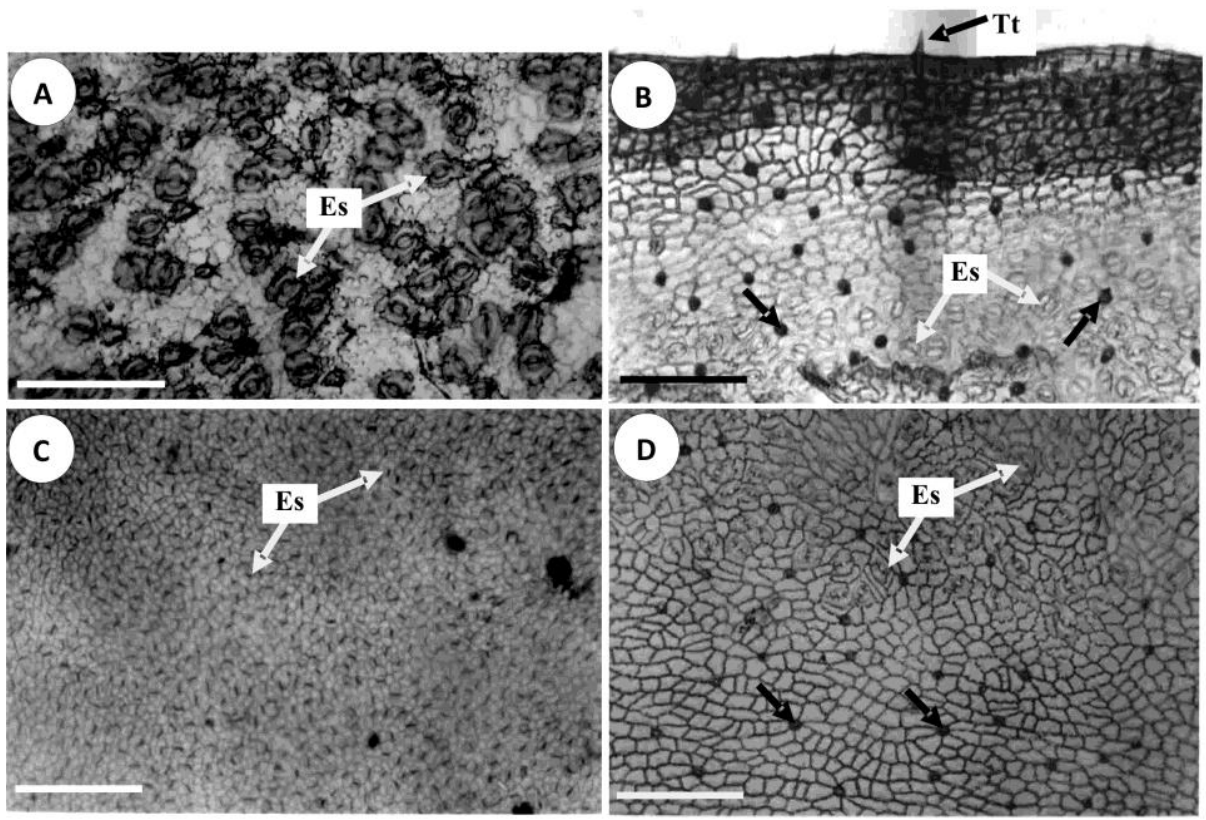

Figura 2. Vista frontal da face abaxial da superfície da lâmina foliar Eugenia L. 2A: E. biflora (L.) DC.; 2B: E. patrisii Vahl.; 2C: E. flavescens DC.; 2D: E. tapacumensis Berg. Es = estômato; $\mathrm{Tt}=$ tricoma tector. Setas $=$ bases de tricomas tectores. Barras $=300 \mu \mathrm{m}$. 
O mesofilo em secção transversal, nas quatro espécies analisadas, é dorsiventral e formado por uma camada de parênquima paliçálido em E. biflora e $E$. tapacumensis, por uma a duas em E. patrisii e por duas a três em E. flavescens (Figura 3). Variações no número de camadas do parênquima paliçádico foram relatadas por (Gomes et al. 2009) em espécies de Myrtaceae oriundas do cerrado. Segundo Esau (1985) e Raven et al. (2007), a estratificação deste tipo de parênquima é influenciada por fatores como intensidade luminosa, disponibilidade de água do solo, umidade atmosférica e posição da folha na planta, não sendo útil para fins de diagnóstico taxonômico.

No parênquima lacunoso das espécies aqui estudadas, ocorrem feixes vasculares colaterais, de pequeno a grande porte, envolvidos por fibras (Figura 3). Organização fibrosa semelhante em torno dos feixes vasculares colaterais foi descrita por Callado (1993) em E. cuprea (Berg.) NDZ.
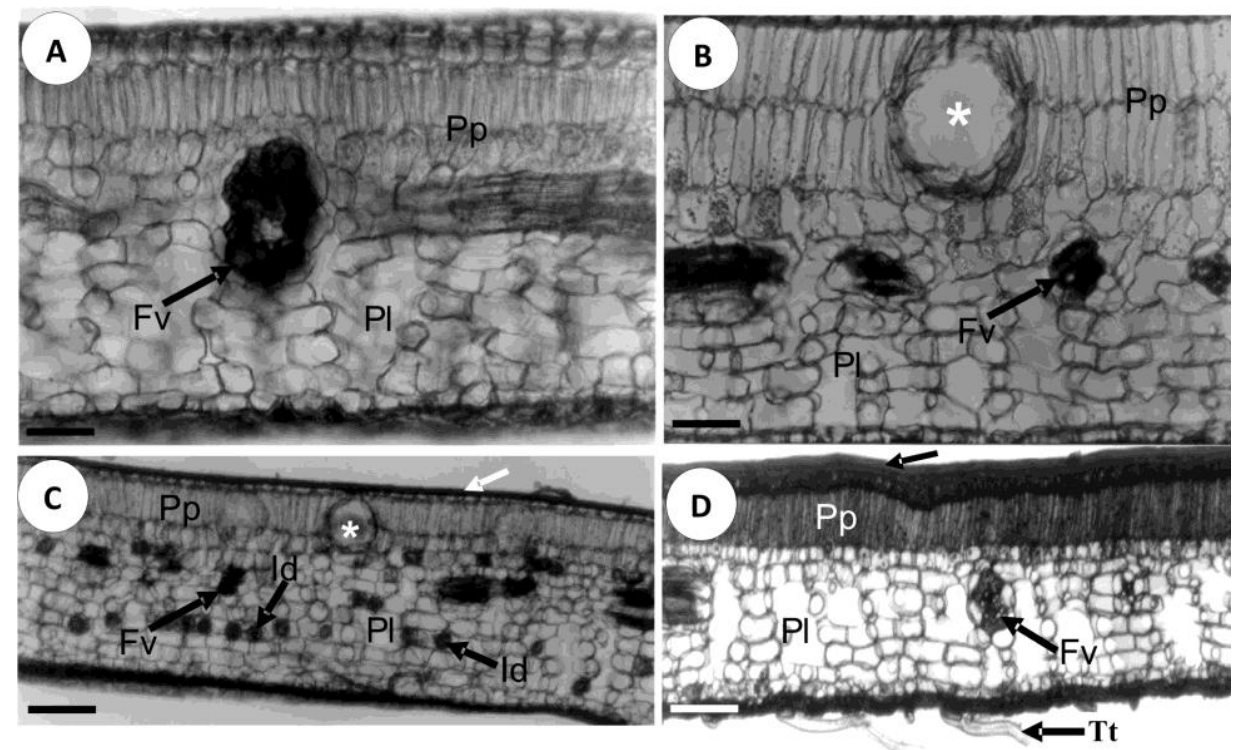

Figura 3. Secções transversais do mesofilo de Eugenia L. 3A: E. patrisii Vahl.; 3B: E. flavescens DC.; 3C: E. tapacumensis Berg; 3D: E. biflora (L.) DC.. Fv = feixe vascular; Id = idioblasto secretor; $\mathrm{Pp}=$ parênquima paliçádico; $\mathrm{Pl}=$ parênquima lacunoso; Asterisco = cavidade secretora; Seta $=$ cutícula. Barras $=200 \mu \mathrm{m}$.

A nervura central em secção transversal, das quatro espécies de Eugenia analisadas, apresenta sistema vascular disposto em arco e formado por um único feixe 
vascular bicolateral, organizado em floema externo, xilema radial e floema interno. Ambos os floemas cerceados por esclerênquima fibroso. (Figura 4).
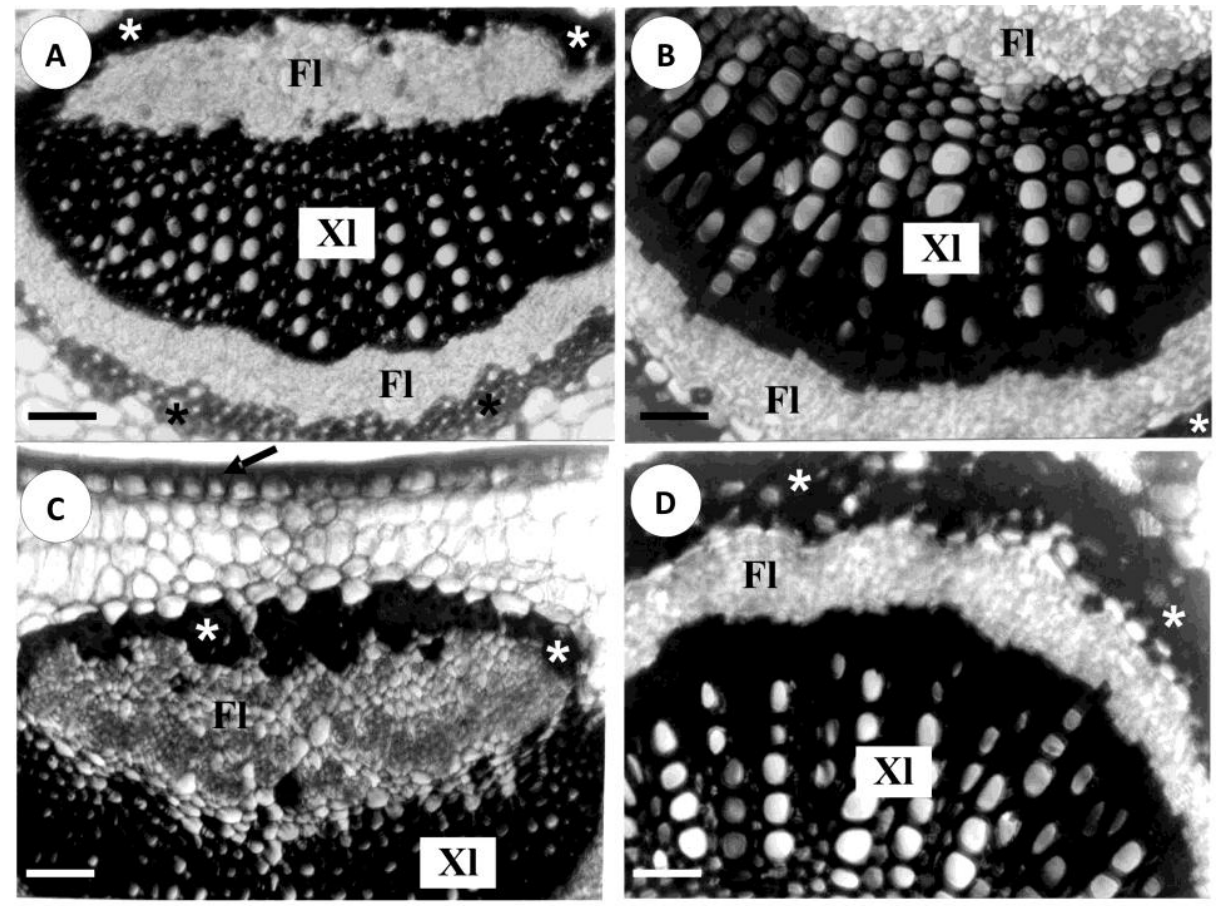

Figura 4. Secções transversais da nervura central de Eugenia L. 4A: E. biflora (L). DC.; 4B: E. flavescens DC.; 4C: E. tapacumensis Berg; 4D: E. patrisii Vahl.. Fl = floema; Xl = xilema; Asterisco $=$ fibras; Seta $=$ cutícula. Barras $=200 \mu \mathrm{m}$.

Espécies de Myrtaceae podem apresentar nervura central com feixe vascular único e em formato de arco (Gomes et al. 2009). Característica esta importante na distinção dos gêneros Psidium L. (Rotman 1976) e Siphoneugena Berg. (Proença 1990) e de espécies de Eugenia (Cardoso \& Sajo 2004). Vascularização bicolateral na nervura central é característica de Myrtales (Vliet \& Baas 1984).

O pecíolo em secção transversal das espécies analisadas possui, na epiderme, inúmeros tricomas tectores unicelulares simples, dispostos de forma isolada ou em tufos. Na região mais central desta estrutura, encontra-se um feixe vascular bicolateral protegido por uma camada de fibras em quase toda sua extensão (Figura 5). Vascularização peciolar semelhante foi descrita por Khatijah et al. (1992) à espécies de 
Eugenia, mas, os autores observaram um sistema de feixes e não um único feixe, bem como a presença de idioblastos com cristais prismáticos de oxalato de cálcio.
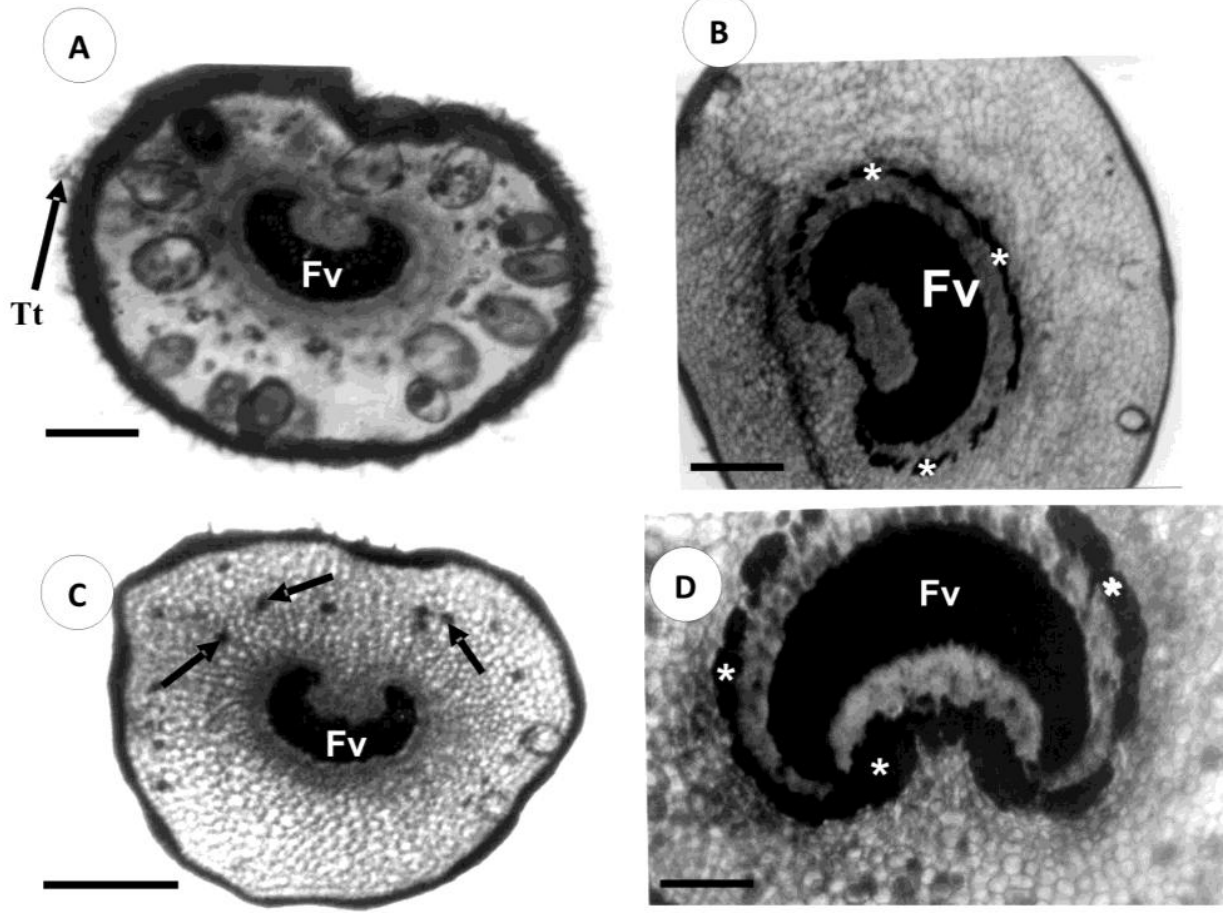

Figura 5. Secções transversais do pecíolo Eugenia L. 5A: E. biflora (L.) DC.; 5B: E. patrisii Vahl.; 5C: E. flavescens DC.; 5D: E. tapacumensis Berg. $\mathrm{Fv}=$ feixe vascular; $\mathrm{Tt}=$ tricoma tector; Asterisco $=$ fibras; Seta $=$ idioblastos secretores. Barras $=200 \mu \mathrm{m}$.

Cavidades secretoras e idioblastos, cristalíferos e secretores, foram observados nas folhas das espécies de Eugenia estudadas. As cavidades ocorrem principalmente no parênquima paliçádico das espécies, notadamente em E. flavescens e E. tapacumensis, exibindo conteúdo positivo para compostos lipídicos (Figuras 3B e 3C). Os idioblastos com cristais prismáticos e em drusas, ambos com reação positiva para oxalato de cálcio, foram verificados apenas nos parênquimas paliçádico de $E$. biflora e lacunoso de E. tapacumensis, respectivamente. Idioblastos secretores apresentaram conteúdo positivo para compostos fenólicos e lipídicos. Os de conteúdo fenólico ocorrem no parênquima lacunoso de E. tapacumensis (Figura 3C) e de preenchimento, cortical e medular, da nervura central das espécies. Os de conteúdo 
lipídico foram verificados no parênquima de preenchimento cortical do pecíolo das espécies (Figura 5C).

Cavidades secretoras, também referidas como glândulas oleíferas, idioblastos secretores e com cristais são características anatômicas ocorrentes em Myrtaceae (Solereder 1908, Metcalfe \& Chalk 1957, Fahn 1979, Esau 1985, Conti et al. 1997). Jorge et al. (2000) descreveram cristais de oxalato de cálcio e idioblastos secretores para E. punicifolia (Humb., Bonpl. \& Kunth) DC. e Donato \& Morretes (2007) identificaram como tanífero, o conteúdo fenólico de E. brasiliensis Lam. Nas espécies de Eugenia aqui estudadas, estudos histoquímicos posteriores são necessários para melhor avaliar in situ os exsudados dos idioblastos secretores.

Secções transversais foliares, das quatro espécies de Eugenia estudadas, revelaram ainda que a epiderme encontra-se revestida por espessa camada cuticular (Figuras 3C, 3D e 4C). A deposição de cutícula em maior ou menor grau está associada ao estresse hídrico (Fahn 1990) e é comum em espécies de Myrtaceae (Arruda \& Fontenelle 1994).

A estrutura anatômica foliar das quatro espécies de Eugenia aqui estudadas apresentou características universais à família Myrtaceae.

\section{Referências}

Arruda, R.C.O.; Fontenelle, G.B. 1994. Contribuição ao estudo da anatomia foliar de Psidium cattleyanum Sabine (Myrtaceae). Revista Brasileira de Botânica 17(1): 25-35.

Auricchio, M.T.; Bacchi, E.M. 2003. Folhas de Eugenia uniflora L. (pitanga): propriedades farmacobotânicas, químicas e farmacológicas. Revista do Instituto Adolfo Lutz 62: 55-61.

Barroso, G.M.; Guimarães, E.F.; Ichaso, C.L.F; Costa, C.G.; Peixoto, A.L. 1978.

Sistemática de Angiospermas do Brasil. São Paulo: Editora da Universidade de São Paulo.

Barroso, G.M.; Peixoto, A.L.; Ichaso, C.L.F.; Guimarães, E.F.; Costa, C.G. 1984.

Sistemática de Angiospermas do Brasil. Viçosa: Imprenssa da UFV-MG. v.2.

Behar, L. 1971. Dados sobre transpiração e anatomia foliar de Eugenia uniflora L.

Ciência e Cultura 23(3): 273-284.

Braga, M.M.N. 1977. Anatomia foliar de Bromeliaceae de Campinas. Acta Amazônica 7(3): 5-74.

Bastos, M.N.C. 1996. Caracterização das formações vegetais na restinga da princesa, Ilha de Algodoal-PA. Tese de Doutorado, Universidade Federal do Pará.

Callado, C.H. 1993-1997. Anatomia foliar de Eugenia cuprea (Berg) NDZ. e Marlierea suaveolens Gard. (Myrtaceae). Rodriguesia 45/49 (71/75): 25-37. 
Cardoso, C.M.V.; Sajo, M.G. 2004. Vascularização foliar e a identificação de espécies de Eugenia L. (Myrtaceae) da bacia hidrográfica do Rio Tibagi, PR. Revista Brasileira de Botânica 27(1): 47-54.

Chamberlain, C.J. 1932. Methods in plant histology. Chicago: University of Chicago Press.

Conti, E.; Litt, A.; Wilson, P.G.; Graham, S.A.; Briggs, B.G.; Johnson, L.A.S.;

Sytsma, K.J. 1997. Interfamilial relationships in Myrtales: molecular phylogeny and patterns of morphological evolution. Systematic Botany 22: 629-647.

Donato, A.M.; Morretes, B.L. 2007. Anatomia foliar de Eugenia brasiliensis Lam.

(Myrtaceae) proveniente de áreas de restinga e de floresta. Revista Brasileira de Farmacognosia 17(3): 426-443.

Donato, A.M.; Morretes, B.L. 2009. Anatomia foliar de Eugenia florida DC.

(Myrtaceae). Revista Brasileira de Farmacognosia 19(3): 759-770.

Esau, K. 1985. Anatomia Vegetal. 3.ed. Barcelona: Omega.

Fahn, A. 1979. Secretory tissues in plants. New York: Academic Press.

Fahn, A. 1990. Plant Anatomy. Oxford: Pergamon Press.

Fontenelle, G.B.; Costa, C.G.; Machado, R.D. 1994. Foliar anatomy and micromorphology eleven species of Eugenia L. (Myrtaceae). Botanical Journal of the Linnean Society 115: 111-133.

Gomes, S.M.A.; Silva, E.A.M.; Lombardi, J.A.; Azevedo, A.A.; Vale, F.H.A. 2005. Anatomia foliar como subsídio à taxonomia de Hippocrateoideae (Celastraceae) no Sudeste do Brasil. Acta Botânica Brasílica 19(4): 945-961.

Gomes, S.M.; Somavilla, N.S.D.N.; Gomes-Bezerra, K.M.; Miranda, S.C.; DeCarvalho, P.S.; Graciano-Ribeiro, D. 2009. Anatomia foliar de espécies de Myrtaceae: contribuições à taxonomia e filogenia. Acta Botânica Brasílica 23(1): 223-238.

Hussin, K.H.; Cutler, D.F.; Moore, D.M. 1992. Leaf anatomical studies of Eugenia L. (Myrtaceae) species from the Malay Peninsula. Botanical Journal of the Linnean Society 110: 137-156.

Joly, A.B. 1985. Botânica. Introdução à Taxonomia Vegetal. 7.ed. São Paulo: Nacional. v.3.

Johansen, D. 1940.Plant microtecnhnique. New York: Mc Graw-Hill Book Company. Johnson, L.A.S.; Briggs, B.G. 1984. Myrtales and Myrtaceae - phylogenetic analysis.

Annals of the Missouri Botanical Garden 71: 700-756.

Jorge, L.I.F.; Aguiar, J.P.L.; Silva, M.L.P. 2000. Anatomia foliar de Pedra-Hume-Caá (Myrcia sphaerocarpa. Myrcia guianensis, Eugenia punicifolia) (Myrtaceae) Acta Amazônica 30(1): 49-57.

Khatijah, H.H.; Cutler, D.R.; Moore, D.M. 1992. Leaf anatomical studies of the Eugenia L. (Myrtaceae) species from the Malay Peninsula Botanical. Botanical Journal of the Linnean Society 110: 137-156.

Landrum, L.R.; Kawasaki, M.L. 1997. The genera of Myrtaceae in Brazil: an illustrated synoptic treatment and identifications keys. Brittonia 49(4): 508-536. 
Leon, H.; Williams, J. 2005. Estudio anatómico de la madera de 21 especies del género Vochysia Poir. (Vochysiaceae). Acta Botánica Venezuelica 28: 213-232.

Lucas, E.J.; Belsham, S.R.; NicLughadha, E.M.; Orlovich, D.A.; Sakuragui, C.M.;

Chase, M.W.; Wilson, P.G. 2005. Phylogenetic patterns in the fleshy-fruited Myrtaceae - preliminary molecular evidence. Plant Systematics and Evolution 251: $35-51$.

Machado, R.D.; Costa, C.G.; Fontanelle, G.B. 1988. Anatomia foliar de Eugenia sulcata Spring ex. Mart. (Myrtaceae) Acta Botânica Brasílica 1(2): supl.275-285.

Medeiros, J.D. 2000. Anatomia foliar de Eugenia umbelliflora Berg (Myrtaceae). Biotemas 13(1): 7-20.

Metcalfe, C.R.; Chalk, L. 1950. Anatomy of Dicotyledons. Oxford: Claredon Press. Metcalfe, C.R.; Chalk. L. 1957. Anatomy of Dicotyledons. Oxford: Claredon Press. Neves, L.J.; Donato, A.M. 1989. Contribuição ao estudo de Eugenia uniflora L. (Myrtaceae). Bradea 5(25): 275-284.

Pereira, A.M.C. 1985. Anatomia foliar de Eugenia nitida Cambess. (Myrtaceae).

Dissertação de Mestrado, Museu Nacional, Universidade Federal do Rio de Janeiro.

Proença, C.E.B. 1990. A revision of Siphoneugena Berg (Myrtaceae, Myrteae). Edinburgh Journal of Botany 47: 239-271.

Raven, H.P.; Evert, F.R.; Eichhorn, E.S. 2007. Biologia vegetal. Rio de Janeiro: Guanabara Koogan.

Rocha, J.E.; Neves, L.J. 2000. Anatomia foliar de Hibiscus tiliaceus L. e Hibiscus pernambucensis Arruda (Malvaceae). Rodriguesia 51(78/79): 113-132.

Rosário, A.S.; Secco, R.S. 2006. Sinopse das espécies de Marlierea Cambess. (Myrtaceae) na Amazônia brasileira. Acta Amazônica 36(1): 37-51.

Rotman, A.D. 1976. Revisión del género Psidium em la Argentina (Myrtaceae). Darwiniana 20: 418-443.

Sajo, M.G.; Rudall, P.J. 2002. Leaf and stem anatomy of Vochysiaceae in relation to subfamilial and suprafamilial systematics. Botanical Journal of the Linnean Society 138: 339-364.

Silveira, M.J.; Harthman, V.C. 2010. Anatomia foliar de Eugenia involucrata DC. e Eugenia bracteata Vell. (Myrtaceae). Insula 39: 47-58.

Solereder, H. 1908. Systematic anatomy of the Dicotyledons. Oxford: Claredon Press.

Sousa, A.V.G. 1971. Contribuição ao estudo da anatomia foliar e da fisiologia de Psidium guayava Raddi. Ciência e Cultura 23(3): 373-382.

Tantawy, M.E. 2004. Morpho-anatomical study on certain taxa of Myrtaceae. Asian Journal of Plant Sciences 3: 274-285.

Vliet, G.J.C.M.; Baas, P. 1984. Wood anatomy and classification of the Myrtales. Annals of the Missouri Botanical Garden 71: 783-800. 
Watson, L.; Dallwitz, M.J. 2007. The families of flowering plants: descriptions, illustrations, identification, and information retrieval. http://delta-intkey.com Version: 1st. Acesso em: 10 abr. 2010.

Wilson, P.G.; O’Brien, M.M.; Heslewood, M.M.; Quinn, C.J. 2005. Relationships within Myrtaceae sensu lato based on a matK phylogeny. Plant Systematic and Evolution 251: 3-19. 